\title{
NEW TYPE OF VOLTAGE AMPLIFIER BASED ON FIELD-EFFECT TRANSISTOR WITH ON-DEMAND AMPLIFICATION FACTOR
}

\author{
B.M. KAMANOV ${ }^{1, *}$, I.B. SAPAEV ${ }^{1}$, F.KH. KHASANOV ${ }^{2}$, G. TURAEVA ${ }^{3}$, D.B. BABAJANOV ${ }^{4}$, L. \\ SUVONOVA $^{1}$, A.S. GANIEV ${ }^{2}$
}

${ }^{I}$ Tashkent Institute of Irrigation and Agricultural Mechanization Engineers, KoriNiyoziy str., Tashkent 100000, Uzbekistan

${ }^{2}$ Tashkent University of Information Technologies named after Muhammad al- Khwarizmi

${ }^{3}$ Tashkent State Agrarian University.

${ }^{4}$ Turin Polytechnic University in Tashkent, Kichik Khalka yuli 17, Tashkent 100095, Uzbekistan

\begin{abstract}
The results of a study of the photosensitivity of the hetero junction Au-vGaAs:O-nCdS-nInP-Au structure under different bias modes are presented. It has been shown experimentally that independently on the surface to be excited, they differ in photosensitivity in the spectral range of 0.85-0.9 $\mu \mathrm{m}$ and 1.31-1.55 $\mu \mathrm{m}$, which is associated with photogeneration processes in high-resistance gallium arsenide and photoemission processes from metal to semiconductor.

KEYWORDS: Hetero Junction, Photosensitivity, Photocurrent, Forward Bias Mode, Integral Light, Monochromatic Radiation
\end{abstract}

Received: Jun 08, 2020; Accepted: Jun 28, 2020; Published: Sep 21, 2020; Paper Id.: IJMPERDJUN20201368

\section{INTRODUCTION}

Recently, more attention of researchers drawn to the problems of the developments and a study of microelectronic devices based on field effect transistors (FET) used to amplify electrical signals in the radio and telecommunication systems. The main task is to increase the gain while maintaining the frequency range and eliminate distortion of the amplified signal. Different approaches are employed to accomplish that goal, perfected by construction and complexity of electronic circuits [1-3].

In field-effect transistors, the value of the output (flowing through the channel) current is controlled by acting on the main charge carriers by the transverse electric field of the input signal. The gain of the FET is determined by the ratio of the outputand the input voltages $K=U_{\text {out }} / U_{\text {in }}$. An amplified voltage is created across a load resistor connected in series to the drain and power supply. To increase the gain coefficient one could use the dynamic resistance (load), for instance a FET, instead of a load resistance.

In the known circuits [4], an active element of the second field-effect transistor is used as the dynamic resistance of the drain load of the field - effect transistor, the internal resistance of which depends on the signal amplitude at the drain of the main transistor. The main transistor is connected according to thecircuit with common source, and the dynamic transistor according to thecircuit of common drain. Channels of both transistors are connected in series, which provides equal currents on them. 
The main disadvantage of this voltage amplifier with dynamic load is the limited amplification factor to 20 and distorted amplification of small signals. In this case, the transistors are in the fixed maximum drain current mode, and not in the economy mode, which contributes to the appearance of thermal noise. Furthermore, with little increase of the input signal $(40 \mathrm{mV})$ the output second signal will be distorted due to the transition of the transistor from the reverse bias mode to the forward bias mode. And when the supply voltage decreases from $9 \mathrm{~V}$ to $6 \mathrm{~V}$, signal distortions begin with an input signal starting from $26 \mathrm{mV}$, although the gain will be $48 \div 50$.

\section{METHODS}

Among the voltage amplifier circuits with a dynamic load an electric circuit of the amplifier [5], which shown in Figure 1 has much interest. In this circuit the load transistor VT1 between source and gate resistance introduced R1, which sets the operating current. In this case, the second transistor is also in the automatic bias mode with resistance R2.

This device also does not allow for high gain of a useful signal and to eliminate distortion of the output signal when input signal level increased, since the gate-source transitions of the first and second transistors are under the different potential. Compared with the known circuits, the presence of resistance between the source and the gate of the second transistor made it possible to increase the gain to 130 .

In the present study we show a new voltage amplifier circuit, with a dynamic load on field-effect transistor, which provides an increase in gain and exclude distortion of the amplifier output signal with dynamical load in comparison to known analogs.

The difference between the proposed scheme [6], Figure 2, is that the gate of the main field-effect transistor (KP303J) is connected to the common terminal of the power supply, and the input capacitor $\mathrm{C} 1$ is connected to the source of the first transistor 1 .

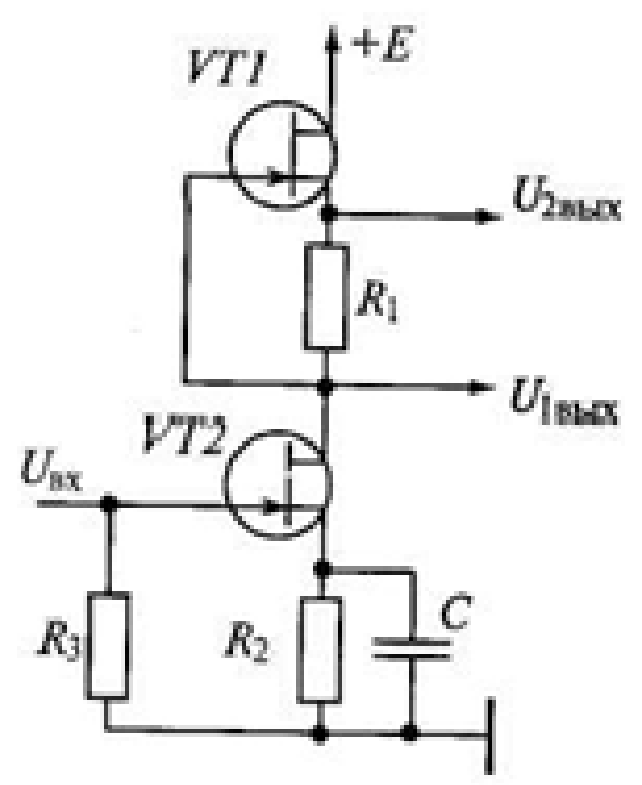




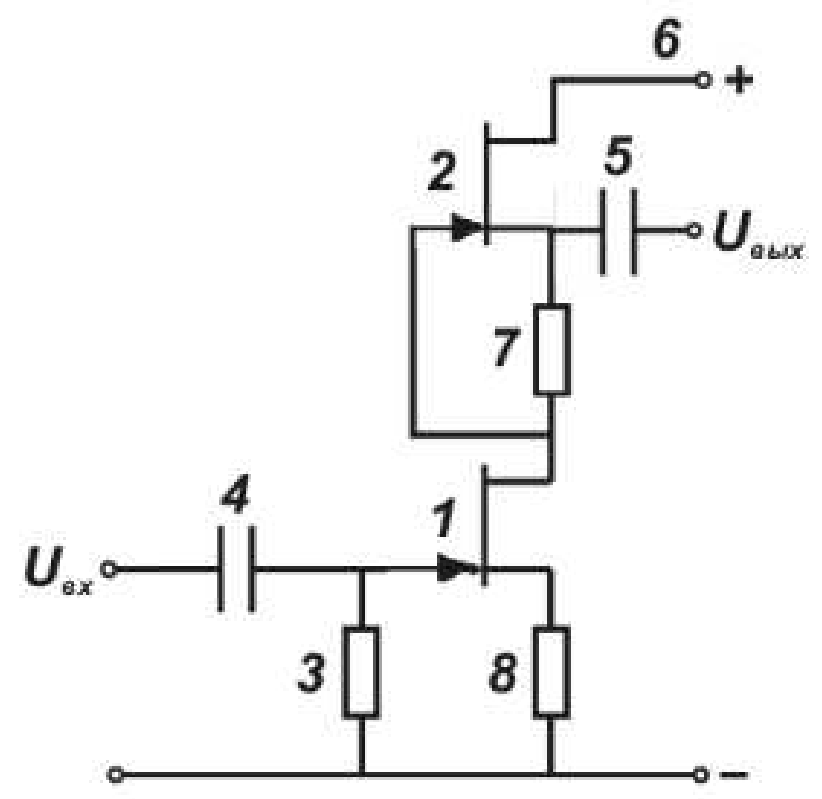

Figure 1: Amplifier with dynamic load

Connection of the gate of the first transistor directly to the minus power supply, to whichthe resistance which contacting the source electrode is also connected, provides, as the second field effect transistor, the bias mode transition of the gate-source through a resistance. As a result, the dynamic output resistanceincreases by contributing to increase of the output voltage.

Submission of the useful signal to the source of the first transistor via a first capacitor 4, instead of the gate, provides deeper modulation of the channel of FET and correspondingly, increases the gain of the field effect transistor.

Providing equal currents of the individual first and second transistors through choosing of the resistance values contribute exclusion of the output waveform distortion, and obtaining a maximum value of the gain.

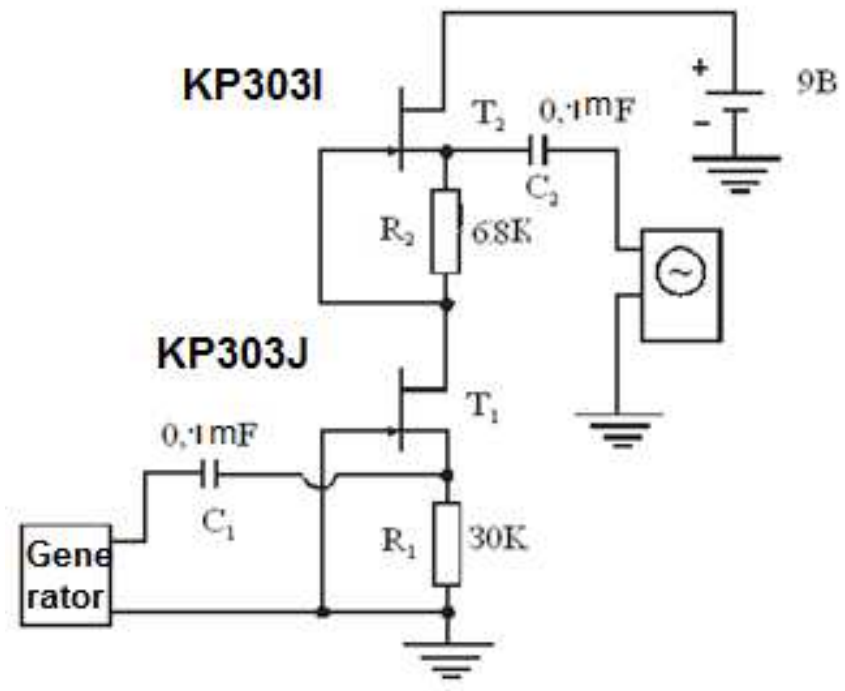

Figure 2 


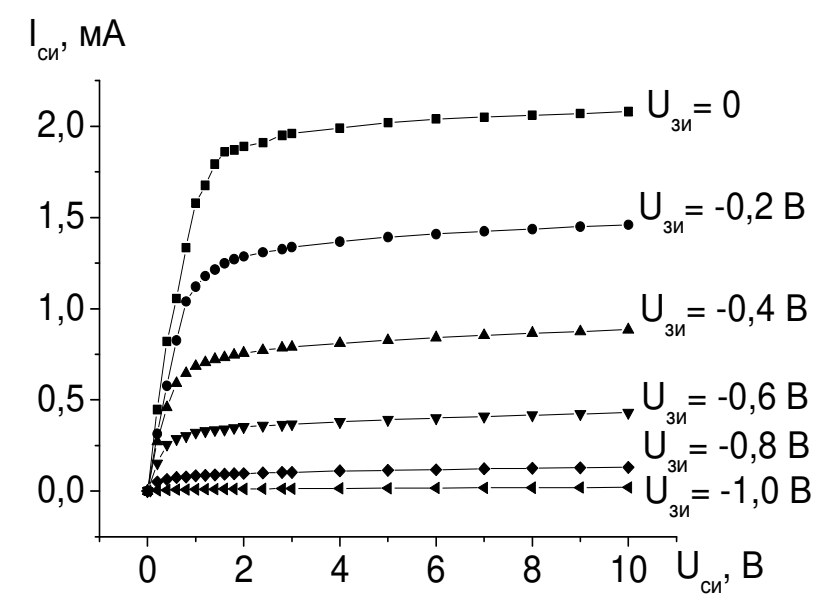

Figure 3: Stock current-voltage characteristic of the field-effect transistor KP303J

As shown in Figure 2, in the proposed series connection of two transistors, the source terminals are connected through a resistance to the gate terminal, and the gate of the first transistor is connected to the source terminal of the second transistor. With respect to the operating voltage, the control p-n junctions are in the blocking mode. As a result, each transistor turns into a two-pole diode and the current of one transistor is equalized by the current of the second transistor, as in two-barrier diode structures [7]. The modulated junction controls the parameters of the second junction by redistributing the voltage applied from an external power source. Since the operating drain currents for both transistors are selected the same, the family of dark drain characteristics in the saturation region remains qualitatively unchanged, Figure 3 , but the saturation voltage increases and the saturation mechanism changes.

The proposed circuit (Figure 2) with a dynamic load is assembled on two field-effect transistors of the 1st type KP303J with a maximum drain current of $1.8 \mathrm{~mA}$ and a cut-off voltage of the channel of $1.0 \mathrm{~V}$ and of the 2nd type KP303I with a maximum drain current of $3.5 \mathrm{~mA}$ and a cut-off voltage of the channel $1.9 \mathrm{~V}$, corresponding to two resistances of 30 $\mathrm{k}$ Ohm and $68 \mathrm{k} \mathrm{Ohm}$. In this case, the measured drain currents of the separately taken first and second transistors are equal to each other. The gate of each field-effect transistor is short-circuited to the source through the indicated resistances, and the drain of the first transistor is connected to the source of the second transistor, and power supply from the 9 volt battery is supplied to the drain terminals of the second transistor and the gate of the first transistor. The useful signal $U_{\text {in }}$ is supplied from the generator of audio signals G3-109 through the 0.1 microfarad capacitor to the source. The output signal is taken from the source of the second field-effect transistor through a $0.1 \mu \mathrm{F}$ capacitor and is recorded with an C1-70 oscilloscope. A useful signal from 1 to $100 \mathrm{mV}$ (at a supply voltage of $9.0 \mathrm{~V}$ ) can be applied to the input.

\section{RESULTS AND DISCUSSIONS}

In a wide frequency range (from 200 to $3000 \mathrm{~Hz}$ ), the resistance of the capacitance $C_{0}$ is large enough and it does not shunt the resistances $R_{c}$ and $R_{3}$, therefore the total resistance in the drain of the transistor is determined by the expression [8,9]

$$
R_{1}=\frac{R_{C} R_{3}}{R_{C}+R_{3}}
$$

The stage gain is 


$$
K=\frac{U_{\text {output }}}{U_{\text {entrance }}}=\frac{I_{C} R_{i}}{U_{\text {entrance }}}=\frac{\mu R_{1}}{R_{1}+R_{i}}=\frac{\mu}{1+\frac{R_{i}}{R_{1}}}=\frac{\mu \alpha}{1+\alpha}
$$

where $I_{\mathrm{c}}$ is a current in the drain of the transistor; $\mu$ is the static gain of the field-effect transistor, $\mu=\Delta U_{\text {drain,source }} /$ $\Delta U_{\text {gate }} ; \alpha=R_{i} / R_{l}$ is a load factor. With $R_{i}>>R_{3}, R_{3}>>R_{c}$ onecanassume $R_{l} / R_{c}>>\left(1+R_{i} / R_{3}\right)$, then the gain in the middle part of the frequency range is

$$
K_{c p}=\frac{\mu}{\frac{R_{i}}{R_{C}}}=S R_{C}
$$

where $S$ is the slope of the field-effect transistor characteristic.

For a given frequency range of $400 \mathrm{~Hz}$ and an input signal of $2 \mathrm{mV}$ with a resistance of $30 \mathrm{k} \Omega$, the gain of a sinusoidal signal equal to 3000 is obtained.

Table 1: Output and gain data at various source-to-gate resistances

\begin{tabular}{|c|l|l|l|l|l|l|l|}
\hline $\mathrm{F}=400 \mathrm{~Hz}, \mathrm{U}_{\text {in }}=2 \mathrm{mV}$ \\
\hline$R_{\mathrm{s}-\mathrm{g}}, \mathrm{k} \Omega$ & 0.43 & 1.0 & 3.0 & 5.0 & 10 & 20 & 30 \\
\hline$U_{\text {out }}, \mathrm{V}$ & 0.8 & 1.0 & 2.0 & 2.4 & 3.2 & 5.0 & 6.0 \\
\hline $\mathrm{K}_{\mathrm{g}}$ & 400 & 500 & 1000 & 1200 & 1800 & 2500 & 3000 \\
\hline
\end{tabular}

The observed increase in the gain with an increase in the resistance of the short-circuiting source to the gate is due to an increase in the thickness of the depletion layer at the source part caused by an increase in the voltage removed from the resistor; as a result, the modulation depth of the base region increases.

\section{CONCLUSIONS}

It is shown experimentally that a voltage amplifier with dynamic load on two field-effect transistors, wherein the gate of each FET is shorted to the source through a resistance, and the drain of the first transistor is connected to the source of the second transistor, and power is supplied to the drain terminal of the second transistor and the gate of the first transistor, the useful signal is fed through a capacitor to the source of the first transistor and is removed through the capacitor from the source of the second transistor, with equal values of the drain currents of each transistor, the maximum value of the gain is obtained without any distortion of the output signal shape.

\section{REFERENCES}

1. Semiconductors: a three-dimensional tomorrow ". http://wiki.miem.edu.ru.

2. Ionescu Adrian M., Riel Heike. Tunnel field_effect transistors as energy_efficient electronic switches // Nature. November 2011. V. 479. № 17. P. 329-337.

3. Kondratyuk A.V. Analysis of the features of typical designs of field-effect transistors with an isolated gate // Young scientist. 2016. - No. 23.- pp. 59-66. - URL https://moluch.ru/archive/127/35054/

4. Jessie Russell. Field-effect transistor, VSD, 2012 .-- 80 p.

5. Voltage amplifier on a field-effect transistor. 
6. http://toe-kgeu.ru/automaticelements/197-automaticelements1

7. Invention patent number IAP 05322 "Voltage amplifier with dynamic load" / Karimov A.V., Yodgorova D.M., Abdulxayev O.A., Kamanov B.M.Bulletin, №12on20.12.2016.

8. Karimov A.V. and Yodgorova D.M. Some Features of Photocurrent Generation in Single and Multibarrier Photodiode Structures. Semiconductors, 2010, Vol. 44, No. 5, pp. 647-652.

9. Low frequency amplifiers based on filed-effect transistors. http://zpostbox.narod.ru/fet_based_ac_amplifiers.html

10. Milekhin A.G. Radio engineering circuits on field-effect transistors. "Energy". Moscow. -1976. -pp. 29-31. 